\title{
О возможности интерференции Рамсея в германии, легированном мелкими примесями
}

\author{
(C) В.В. Цыпленков, В.Н. Шастин
}

Институт фризики микроструктур РАН - филиал Федерального государственного бюджетного научного учреждения „Федеральный исследовательский центр Институт прикладной физики Российской академии наук“, 603087 Нижегородская обл., Россия

E-mail: Tsyplenkov1@yandex.ru

Поступила в Редакцию 15 апреля 2020 г.

В окончательной редакции 21 апреля 2020 г.

Принята к публикации 21 апреля 2020 г.

Теоретически рассмотрена динамика образования и распада когерентных состояний мелких примесных центров в кристалле германия, резонансно-возбуждаемых парой лазерных импульсов, следующих друг за другом с некоторой задержкой. Сделаны оценки необходимой мощности излучения, температуры кристаллической решетки кристалла и предельно допустимых неоднородных ширин примесных линий для наблюдения интерференции Рамсея в такой системе.

Ключевые слова: германий, мелкие доноры, лазерное возбуждение, когерентные состояния, интерференция Рамсея.

DOI: $10.21883 /$ FTP.2020.08.49630.06

\section{1. Введение}

В настоящее время научная активность многих исследовательских групп направлена на исследование квантовых систем с целью создания на их основе принципиально новых устройств, сопряженных с развитой кремниевой и германиевой технологией. В качестве кандидатов, в частности, рассматриваются кулоновские центры в полупроводниках [1-6]. Интерес вызывает создание квантовых затворов [2], одноатомных транзисторов [3], изучение механизмов управления одними центрами легирования (например, доноров) посредством влияния на возбужденные состояния соседних атомов (дипольная блокада ридберговских центров), эффектов когерентного излучения на внутрицентровых переходах и создание платформы для кубитов на основе донорных центров в полупроводниках [5-9]. Одним из способов оптического управления квантовым состоянием системы (создание состояния и его „считывание“) является метод интерферометрии Рамсея [10], использующий импульсное оптическое возбуждение. Суть явления заключается в том, что населенность верхнего уровня двухуровневой системы после воздействия последовательности двух оптических импульсов возбуждения с частотой, близкой к частоте перехода, осцилляторно зависит от разности фаз излучения в первом и втором импульсах. Таким образом, изменяя разность фаз, например, изменяя с помощью оптической линии задержки временной интервал между импульсами, можно контролируемым образом создавать любую квантовую суперпозицию состояний. Другим важным аспектом является возможность некогерентного „считывания“ квантового состояния системы в полупроводниках. Ярким результатом в этом направлении является работа [11], в которой наблюдались осцилляции Рамсея (Ramsey fringes) при когерентном возбуждении доноров фосфора в кремнии импульсным излучением лазера на свободных электронах (FELIX, Netherlands) и продемонстрирована возможность электрического „считывания“ квантового состояния системы посредством регистрации фототока, обусловленного термической ионизацией доноров с верхнего возбуждаемого уровня донора фосфора.

Мелкие доноры в германии обладают целым рядом преимуществ с точки зрения возможности создания полупроводниковых кубитов. К таковым можно отнести большие радиусы донорных возбужденных состояний, снижающие требования к технологии в случае использования планарной архитектуры Кейна, значительные величины матричных элементов дипольного момента, а также большие величины времен жизни $T 1$ возбужденных состояний доноров (до 1 нс и более $[12,13]$ ), определяющие предельно достижимое время поперечной релаксации $T 2$. Создание и считывание когерентных суперпозиций, в частности, возможно с помощью источников когерентного излучения. В этом контексте стоит упомянуть возможность создания источников стимулированного излучения на основе внутрицентровых переходов доноров германия [14], что позволит создавать управляющий источник излучения и кубиты с использованием единой полупроводниковой базы.

В настоящей работе проводится анализ возможности реализации при помощи интерферометрии Рамсея управляемых когерентных состояний мелких доноров в кристалле германия. В двухуровневом приближении теоретически рассмотрено взаимодействие донорного центра с последовательностью двух импульсов лазерного излучения, находящихся в резонансе с какимлибо разрешенным в электродипольном приближении переходом между состояниями донора. 


\section{2. Теория и выводы}

Теоретическое описание строилось в рамках полуклассической модели, в которой примесный атом считался двухуровневой квантовой системой, электромагнитное поле же (два последовательных импульса электромагнитного излучения на частоте, близкой к частоте атомного перехода с произвольным фазовым сдвигом) описывалось классически. Использовалось приближение вращающейся волны. Описание взаимодействия атома с полем в данном случае удобно строить, используя метод амплитуд вероятности (см., например, [15]), так как он позволяет избежать при численных вычислениях работы с быстроосциллирующими функциями, как в методе матрицы плотности (недиагональные элементы), и позволяет не увеличивать кратность системы дифференциальных уравнений, как в методе уравнений Блоха в случае, когда увеличивается число импульсов излучения или число атомных уровней. Гамильтониан системы в пренебрежении взаимодействием с колебаниями решетки имеет вид

$$
H=H_{0}+\mu E_{1}(t) \cos (v t)+\mu E_{2}(t-\tau) \cos (v t+\varphi),
$$

где $H_{0}$ - гамильтониан невозмущенной системы, собственные функции которого $\varphi_{1}$ и $\varphi_{2}$ с энергиями $\hbar \omega_{1}$ и $\hbar \omega_{2}, \mu$ - дипольный момент перехода, $E_{1,2}(t)-$ зависящие от времени амплитуды полей двух импульсов внешнего излучения, $v$ - круговая частота этого излучения, $\varphi-$ разность фаз излучения в первом и втором импульсах, $\tau$ - временна́я задержка между двумя импульсами. Волновую функцию в рамках такого описания можно записать в виде

$$
\Psi(t)=a_{1}(t) \varphi_{1} e^{-i \omega_{1} t}+a_{2}(t) \varphi_{2} e^{-i \omega_{2} t},
$$

где $a_{1}(t), a_{2}(t)$ - искомые амплитуды. Подставляя волновую (2) в гамильтониан (1), после несложных преобразований можно получить систему уравнений для $a_{1}(t)$ и $a_{2}(t)$ :

$$
\left\{\begin{array}{l}
a_{1}^{\prime}=-\frac{i}{2} a_{2}\left(\Omega_{1}(t) e^{i \delta t}+\Omega_{2}(t-\tau) e^{i(\delta t+\varphi)}\right), \\
a_{2}^{\prime}=-\frac{i}{2} a_{1}\left(\Omega_{1}(t) e^{-i \delta t}+\Omega_{2}(t-\tau) e^{-i(\delta t+\varphi)}\right),
\end{array}\right.
$$

где $\Omega_{1}$ и $\Omega_{2}$ - зависящие от $t$ частоты Раби для полей, связанных с первым и вторым импульсами соответственно и определяемые выражениями:

$$
\Omega_{1,2}(t)=\frac{\mu_{21} E_{1,2}(t)}{\hbar} .
$$

Здесь $\delta$ - отстройка частоты излучения от частоты атомного перехода, $\mu_{21}$ - матричный элемент дипольного момента на переходе между рассматриваемыми уровнями атома. Форма импульсов излучения (следовательно, $\Omega_{1}$ и $\Omega_{2}$ ) аппроксимировалась гауссовой кривой. Учет неоднородного уширения в среде производился усреднением по $\delta$ найденных $a_{1}(t)$ и $a_{2}(t)$ и величин, производных от них, считая, что $\delta$ - случайная величина с нормальным распределением.

Решая систему уравнений (3), можно построить зависимость населенности верхнего уровня $\left|a_{2}\right|^{2}$ от разности фаз $\varphi$. Эта зависимость будет иметь синусоидальную форму (осцилляции Рамсея (Ramsey fringes)) с амплитудой и фазовой постоянной $\varphi_{0}$, которые сложным образом зависят от отстройки $\delta$ и формы импульсов $\Omega_{1}(t)$ и $\Omega_{2}(t)$ (а при заданной форме от их длительности $T$ ).

Можно ввести величину площади импульса, определяемую выражением

$$
\int \Omega_{1,2}(t) d t=\overline{\Omega_{1,2}} T
$$

где $\overline{\Omega_{1,2}}$ - средние по времени значения частоты Раби в течение действия оптического импульса возбуждения. По значению этой площади происходит классификация импульсов. Оптимальным для проведения рамсейинтерферометрии является, когда площадь импульса равняется $\pi / 2$, такой импульс в литературе называется $\pi / 2$-импульсом. Такой импульс приводит систему на экватор сферы Блоха. Это накладывает условия на мощность излучения в импульсах возбуждения. Используя известные электродинамические соотношения [см., например, 16], не трудно получить выражение для средней интенсивности импульсов (в системе СГСЕ):

$$
\bar{S}=\frac{\pi c \sqrt{\varepsilon}}{32}\left(\frac{\hbar}{\mu_{21} T}\right)^{2},
$$

где $\varepsilon$ - диэлектрическая проницаемость среды, $c-$ скорость света. Матричный элемент дипольного момента перехода $\mu_{21}$ не трудно получить из экспериментальных линий поглощения на переходах между состояниями донорных центров в германии, используя соотношение (в системе СГСЕ) [17]:

$$
\mu_{21}^{2}=\frac{\hbar c \varepsilon}{4 \pi^{2} v_{12}} \int \sigma d v
$$

где $v_{12}$ - центральная частота линии перехода, $\sigma$ сечение перехода, зависящее от частоты $v$. Используя это соотношение, можно вычислить, что, например, для донора мышьяка в германии значение $\mu_{21} / e$ равняется $\sim 8 \AA$ для перехода $2 p_{0}-1 s(A)$ и $\sim 17 \AA$ для перехода $2 p_{ \pm}-1 s(A)$. На рис. 1 представлены вычисленные интенсивности излучения в импульсах возбуждения, необходимые для осуществления интерферометрии Рамсея в зависимости от их длительности $T$ (определяются источником излучения) для переходов $2 p_{0}-1 s(A)$ и $2 p_{ \pm}-1 s(A)$ в донорах мышьяка в германии. Существующие источники, такие как ЛСЭ „FELIX, Netherlands“, „FELBE, Germany“, „NovoFEL, Новосибирск“, с большим запасом обеспечивают такие значения интенсивности. 
На рис. 2 показаны зависимости амплитуды $\left|a_{2}\right|^{2}(\varphi)$ при различных $\delta$. При увеличении $\delta$ происходит не только уменьшение амплитуды осцилляций, но и изменяется фазовый сдвиг $\varphi_{0}$. Это приводит к тому, что при усреднении $\left|a_{2}\right|^{2}$ по $\delta$ в реальной среде (учет неоднородного уширения линии перехода) амплитуда осцилляций стремительно падает с увеличением неоднородной ширины линии $\Lambda$. Понижая концентрацию легирования и используя изотопически чистый германий, можно уменьшить неоднородное уширение линий, и ширина примесных линий приблизится к естественной ширине, определяемой скоростью внутрицентровой релаксации состояний примеси с излучением фононов. Согласно расчетам [14,18], характерная скорость внутрицентровых переходов между верхними возбужденными состояниями со спонтанным излучением фонона $w_{c n} \sim 2 \cdot 10^{9} \mathrm{c}^{-1}$, что соответствует экспериментальной оценке темпов релаксации, полученным методом „рump-probe“ [13]. Таким образом, предельно достижимая ширина примесных линий стремится к значению $w_{c n} / 2 \pi \sim 0.0012$ мэВ.

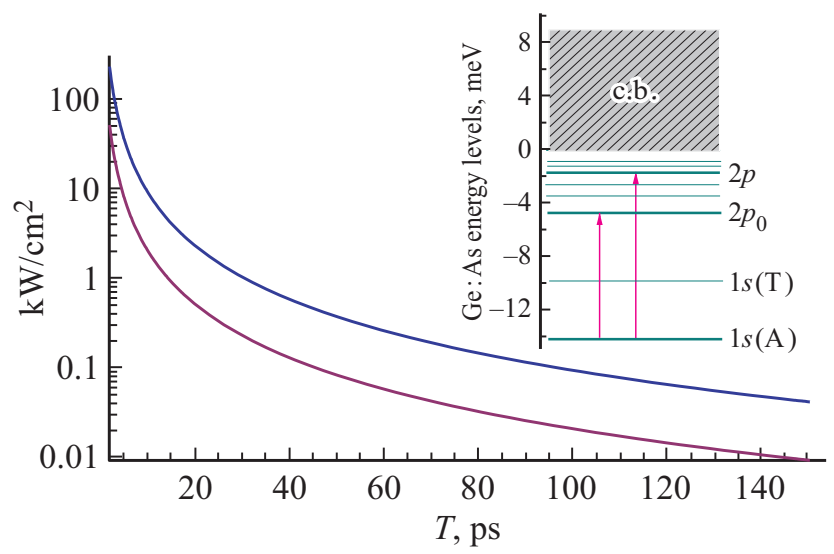

Рис. 1. Зависимость интенсивности излучения $\pi / 2$-импульса возбуждения для переходов $1 s(A)-2 p_{0}$ (верхняя кривая) и $1 s(A)-2 p_{ \pm}$(нижняя кривая) в донорах Аs в германии от его длительности. На вставке - схема уровней донора As в Ge.

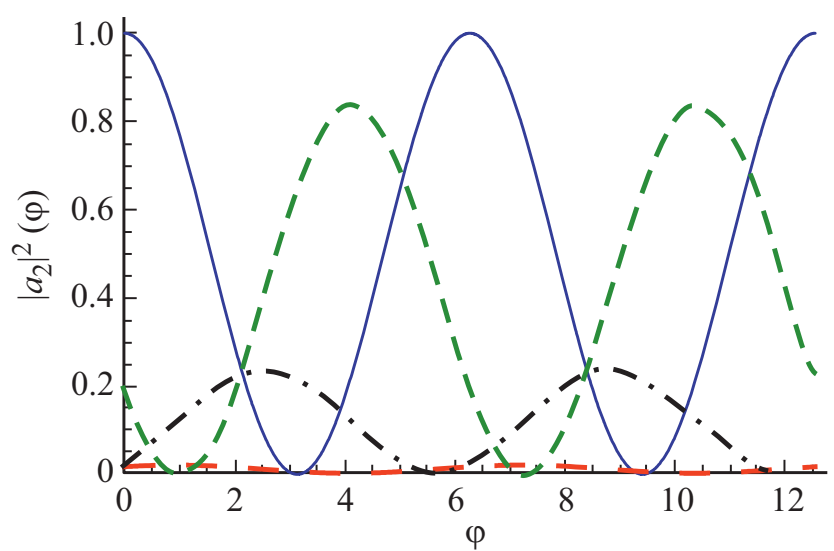

Рис. 2. Зависимость $\left|a_{2}\right|^{2}(\varphi)$ при длительности импульсов возбуждения $T=100$ пс (измеренной на полувысоте) при различных величинах отстройки $\delta=0,0.02,0.04$ и 0.06 мэВ в порядке уменьшения амплитуды осцилляций.

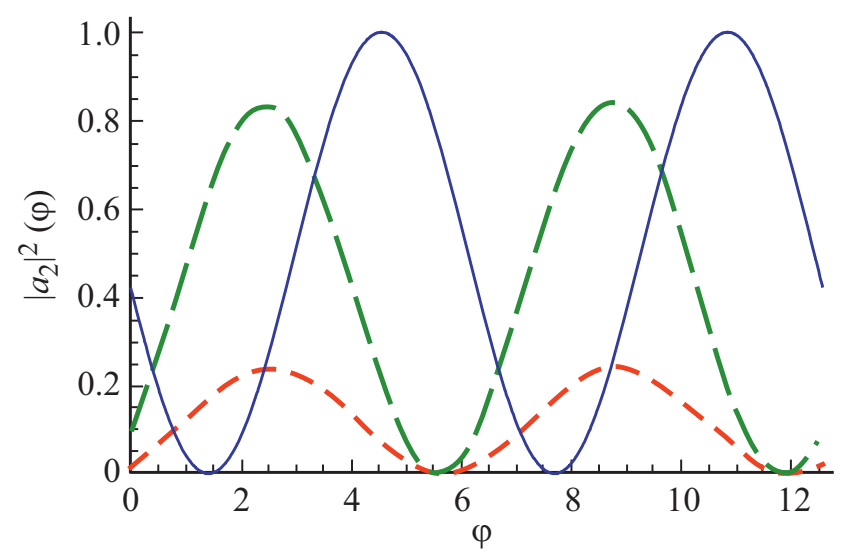

Рис. 3. Зависимость $\left|a_{2}\right|^{2}(\varphi)$ при различных длительностях импульсов возбуждения $T=10,50,100$ пс в порядке уменьшения амплитуды осцилляций при величине отстройки $\delta=0.04$ мэВ (при изменении $T$ изменялись и амплитуды импульсов возбуждения так, чтобы они оставались $\pi / 2$-импульсами).

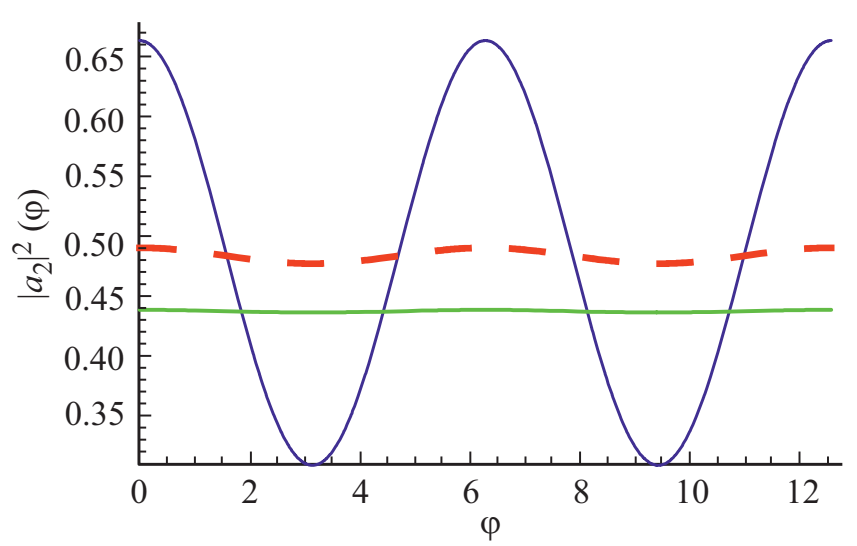

Рис. 4. Зависимость $\left|a_{2}\right|^{2}(\varphi)$ при неоднородном уширении линии перехода $\Delta=0.05$ мэВ при различных длительностях импульсов возбуждения $T=10,20,50$ пс в порядке уменьшения амплитуды осцилляций.

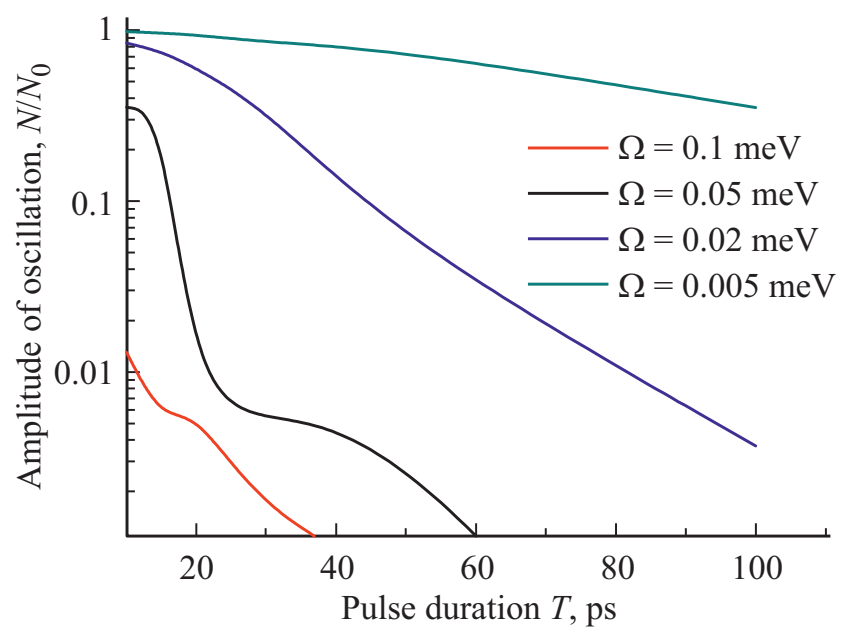

Рис. 5. Зависимость амплитуды осцилляций $\left|a_{2}\right|^{2}(\varphi)$ от $T$ при некоторых величинах неоднородного уширения в среде. При изменении $T$ изменялись и амплитуды импульсов возбуждения так, чтобы они оставались $\pi / 2$-импульсами. 


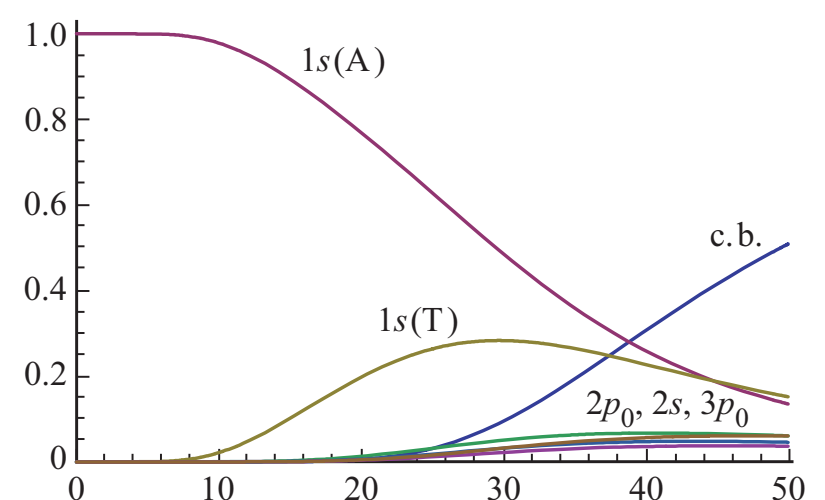

Рис. 6. Равновесные населенности состояний донора мышьяка в германии (с учетом кратности вырождения уровней) в зависимости от температуры решетки кристалла, рассчитанные в соответствии с распределением Больцмана.

Результат вычислений также показывает интересную зависимость от длительности импульса возбуждения $T$ : чем меньше $T$, тем больше амплитуда осцилляций при заданных $\delta$ (либо $\Delta$ ), тем больше, следовательно, шансов наблюдать эффект экспериментально. Последнее демонстрируют рис. 3-5, на которых отображен характер зависимостей от длительности импульсов $T$ амплитуды осцилляций Рамсея $\left(\left|a_{2}\right|^{2}(\varphi)\right)$ при некоторых значениях $\delta$ и $\Delta$. Из рис. 5 видно, что зависимость амплитуды осцилляций имеет сугубо нелинейную зависимость от длительности импульсов возбуждения $T$. Как предполагается, чтобы осцилляции Рамсея были наблюдаемы в эксперименте, необходимо, чтобы населенность верхнего уровня $\left|a_{2}\right|^{2}$ изменялась на десятки процентов при изменении $\varphi$.

Важным аспектом для наблюдения различных когерентных эффектов в полупроводниковых средах является учет взаимодействия с колебаниями решетки. Взаимодействие с фононами приводит к нарушению когерентности в системе и является одной из причин температурного ограничения для наблюдения интерференции Рамсея в германии, легированном мелкими донорами. При конечной температуре решетки кристалла происходит увеличение фононных чисел заполнения в соответствии с распределением Бозе-Эйнштейна и, следовательно, увеличение темпов внутрицентровых переходов. Температура, при которой фононные числа заполнения достигают значений $\sim 1$, происходит двукратное увеличение внутрицентровых темпов переходов. И ее можно взять за оценку, при которой происходит существенное уменьшение возможности наблюдения интерференции Рамсея в рассматриваемой системе. Учитывая характерные энергии внутрицентровых переходов между состояниями $2 p_{0} / 2 p_{ \pm}$(соответствуют наиболее сильным линиям в спектре поглощения в германии, легированном мелкими донорами $(\mathrm{Sb}, \mathrm{As}, \mathrm{P}))$ и другими возбужденными состояниями, а также характерную скорость спонтанного излучения фононов $\left(\sim 2 \cdot 10^{9} \mathrm{c}^{-1}\right)$, в качестве оценки такой температуры можно получить значение $\sim 30 \mathrm{~K}$. Приблизительно при такой же температуре происходит существенное уменьшение населенности основного состояния мелких доноров мышьяка с равновесным заселением более высоколежащих уровней и континуума состояний. На рис. 6 представлена рассчитанная зависимость от температуры населенностей состояний донора мышьяка в германии, считая, что носители распределены по энергетическим уровням донорного центра в соответствии с распределением Больцмана.

\section{3. Заключение}

Проведенный в настоящей работе анализ возможности наблюдения интерференции Рамсея в германии, легированном мелкими донорами V группы ( $\mathrm{Sb}, \mathrm{As}, \mathrm{P})$, дает оценки необходимых для этого условий. Вычислены интенсивности излучения в импульсах возбуждения, необходимые для того, чтобы обеспечить оптимальную наблюдаемость интерференции Рамсея на мелких доноpax V группы в германии. Показано, что для наблюдения эффекта необходимо обеспечить температуру решетки, не превышающую 30 К. Допустимые неоднородные уширения линий перехода, определяемые в первую очередь концентрацией легирования, определяют максимальные длительности импульсов возбуждения. Показано, что при величине неоднородного уширения $\sim 0.1$ мэВ необходимо, чтобы длительность импульсов не превышала 10 пс. Источниками с такой длительностью импульсов являются лазеры на свободных электронах FELIX (Нидерланды) и FELBE (Дрезден-Россендорф). При длительности импульсов $\sim 100$ пс (такие импульсы дает Новосибирский ЛСЭ NovoFEL) необходимо, чтобы ширина примесной линии рассматриваемого перехода была близка к естественной ширине линии, равной нескольким $\mu$ Э.

\section{Финансирование работы}

Работа выполнена при поддержке Российского научного фонда (№ 19-72-20163).

\section{Конфликт интересов}

Авторы заявляют, что у них нет конфликта интересов.

\section{Список литературы}

[1] Pla, J.J.K.Y. Tan, J.P. Dehollain, W.H. Lim, J.J.L. Morton, F.A. Zwanenburg, D.N. Jamieson, A.S. Dzurak, A. Morello. Nature, 496, 334 (2013).

[2] A.M. Stoneham, A.J. Fisher, P.T. Greenland. J. Phys. Condens. Matter, 15, L447 (2003).

[3] M. Fuechsle, J.A. Miwa, S. Mahapatra, H. Ryu, S. Lee, O. Warschkow, L.C.L. Hollenberg, G. Klimeck, M.Y. Simmons. Nature Nanotechnology, 7, 242 (2012).

[4] L.C.L. Hollenberg, C.J. Wellard, C.I. Pakes, A.G. Fowler. Phys. Rev. B, 69, 233301 (2004). 
[5] A.P. Heberle et al. IEEE J. Sel. Top. Quant. Electron, 2, 769775 (1996).

[6] K.J. Morse et al. Science Adv. / Quant. Phys., 3, e1700930 (2017).

[7] K.A. Kovalevsky, N.V. Abrosimov, R.Kh. Zhukavin, S.G. Pavlov, H.-W. Hübers, V.V. Tsyplenkov, V.N. Shastin. Quant. Electron., 45, 113 (2015).

[8] S.G. Pavlov, H.-W. Hübers, N. Deßmann, V.N. Shastin, R.K. Zhukavin, B. Redlich, A.F.G. van der Meer, M. Mittendorff, S. Winnerl, N.V. Abrosimov, H. Riemann. Phys. Rev., X, 021009 (2014).

[9] S.G. Pavlov, H.-W. Hübers, R.K. Zhukavin, V.N. Shastin. Phys. Status Solidi B, 250, 9-36 (2013).

[10] N.F. Ramsey. Phys. Rev., 78, 695 (1950).

[11] K.L. Litvinenko, E.T. Bowyer, P.T. Greenland, N. Stavrias, Juerong Li, R. Gwilliam, B.J. Villis, G. Matmon, M.L.Y. Pang, B. Redlich, A.F.G. van der Meer, C.R. Pidgeon, G. Aeppli, B.N. Murdin. Nature Commun., 6, 7549 (2015).

[12] Р.Х. Жукавин, К.А. Ковалевский, С.М. Сергеев, Ю.Ю. Чопорова, В.В. Герасимов, В.В. Цыпленков, Б.А. Князев, Н.В. Абросимов, С.Г. Павлов, В.Н. Шастин, Г. Шнайдер, Н. Дессманн, О.А. Шевченко, Н.А. Винокуров, Г.Н. Кулипанов, Г.-В. Хьюберс. Письма ЖЭТФ, 106 (9), 555 (2017).

[13] Р.Х. Жукавин, К.А. Ковалевский, Ю.Ю. Чопорова, В.В. Цыпленков, В.В. Герасимов, П.А. Бушуйкин, Б.А. Князев, Н.В. Абросимов, С.Г. Павлов, Г.-В. Хьюберс, В.Н. Шастин. Письма ЖЭТФ, 110, 677 (2019).

[14] В.В. Цыпленков, В.Н. Шастин. ФТП, 52, 1469 (2018).

[15] М.О. Скалли, М.С. Зубайри. Квантовая оптика (М., Физматлит, 2003).

[16] Л.Д. Ландау, Е.М. Лифшиц. Электродинамика сплошных сред (М., Наука, 1982) с. 395.

[17] О. Звелто. Принципы лазеров (М., Мир, 1990).

[18] В.В. Цыпленков, В.Н. Шастин. ФТП, 53, 1372 (2019).

Редактор А.Н. Смирнов

\title{
On the possibility of Ramsey interference in germanium doped with shallow impurities
}

\author{
V.V. Tsyplenkov, R.Kh. Zhukavin, V.N. Shastin
}

Institute for Physics of Microstructures

of the Russian Academy of Sciences, 603087 Nizhny Novgorod region, Russia

\footnotetext{
Abstract Dynamics of creation and distraction of coherent states resonantly excited by pair of laser pulses following each other with temporal delay in germanium crystal doped with shallow impurities has been theoretically considered. The power of excited radiation, lattice temperature and limit permissible of inhomogeneous line broadening that necessary to observe of Ramsey interference in such system have been estimated.
} 